The Mathematical Legacy of Srinivasa Ramanujan 
M. Ram Murty • V. Kumar Murty

\section{The Mathematical Legacy of Srinivasa Ramanujan}

曾 Springer 


\author{
M. Ram Murty \\ Department of Mathematics and Statistics \\ Queen's University \\ Kingston, Ontario \\ Canada
}

\author{
V. Kumar Murty \\ Department of Mathematics \\ University of Toronto \\ Toronto, Ontario \\ Canada
}

ISBN 978-81-322-0769-6

ISBN 978-81-322-0770-2 (eBook)

DOI 10.1007/978-81-322-0770-2

Springer New Delhi Heidelberg New York Dordrecht London

Library of Congress Control Number: 2012949894

(C) Springer India 2013

This work is subject to copyright. All rights are reserved by the Publisher, whether the whole or part of the material is concerned, specifically the rights of translation, reprinting, reuse of illustrations, recitation, broadcasting, reproduction on microfilms or in any other physical way, and transmission or information storage and retrieval, electronic adaptation, computer software, or by similar or dissimilar methodology now known or hereafter developed. Exempted from this legal reservation are brief excerpts in connection with reviews or scholarly analysis or material supplied specifically for the purpose of being entered and executed on a computer system, for exclusive use by the purchaser of the work. Duplication of this publication or parts thereof is permitted only under the provisions of the Copyright Law of the Publisher's location, in its current version, and permission for use must always be obtained from Springer. Permissions for use may be obtained through RightsLink at the Copyright Clearance Center. Violations are liable to prosecution under the respective Copyright Law.

The use of general descriptive names, registered names, trademarks, service marks, etc. in this publication does not imply, even in the absence of a specific statement, that such names are exempt from the relevant protective laws and regulations and therefore free for general use.

While the advice and information in this book are believed to be true and accurate at the date of publication, neither the authors nor the editors nor the publisher can accept any legal responsibility for any errors or omissions that may be made. The publisher makes no warranty, express or implied, with respect to the material contained herein.

Printed on acid-free paper

Springer is part of Springer Science+Business Media (www.springer.com) 
On a height he stood that looked towards greater heights.

Our early approaches to the Infinite Are sunrise splendours on a marvellous verge While lingers yet unseen the glorious sun. What now we see is a shadow of what must come.

Sri Aurobindo, Savitri 1.4

How I wish I could show you the world through my eyes.

Vivekananda 


\section{Preface}

22 December 2012 marks the 125th birth anniversary of the Indian mathematician Srinivasa Ramanujan. Being largely self-taught, he emerged from extreme poverty to become one of 20th century's most influential mathematicians. His story is a phenomenal "rags to mathematical riches" story. In his short life, he had a wealth of ideas that have transformed and reshaped 20th century mathematics. These ideas continue to shape mathematics of the 21 st century.

This book is meant to be a panoramic view of his essential mathematical contributions. It is not an encyclopedic account of Ramanujan's work. Rather, it is an informal account of some of the major developments that emanated from his work in the 20th and 21st centuries. The twelve essays focus on a subset of his significant papers and show how these papers shaped the course of modern mathematics.

These essays are based on lectures given by the authors over the years at the Chennai Mathematical Institute, Harish-Chandra Research Institute, IISER (Kolkata), IISER (Bhopal), IIT (Powai), IIT (Chennai), Institute for Mathematical Sciences (Chennai), and the Tata Institute for Fundamental Research (Mumbai) as well as Queen's University, the Fields Institute, and the University of Toronto. The lectures were given so that the material is accessible to undergraduates and graduate students. We have striven to not be too technical. At the same time, we tried to convey some depth of the mathematical theories emerging from the work of Ramanujan. Surely, it is impossible to be comprehensive in such a mammoth task. Still, we hope that the reader will see how the vast landscape of Ramanujan's garden has blossomed over the past century.

Toronto, Canada

M. Ram Murty

V. Kumar Murty 


\section{Contents}

1 The Legacy of Srinivasa Ramanujan $\ldots \ldots \ldots \ldots$

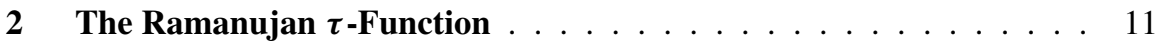

1 Introduction ................... 11

2 The $\tau$-Function and Partitions . . . . . . . . . . . . 12

3 Related Generating Functions . . . . . . . . . . . . . . . . . 13

4 Values of the $\tau$-Function . . . . . . . . . . . . . . . . 14

5 Parity of the $\tau$-Function . . . . . . . . . . . . . 17

6 Congruences Satisfied by the $\tau$-Function . . . . . . . . . 17

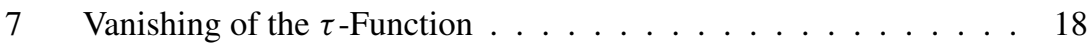

8 Divisibility of $\tau(p)$ by $p \ldots \ldots \ldots \ldots \ldots$

9 Lehmer's Conjecture and Harmonic Weak Maass Forms . . . . . . 21

3 Ramanujan's Conjecture and $\ell$-Adic Representations _ . . . . . . 25

1 The Weil Conjectures . . . . . . . . . . . . . . . . . 26

2 The Case of Elliptic Curves . . . . . . . . . . . . . . . 28

$3 \quad \ell$-Adic Representations . . . . . . . . . . . . . . . . . . 30

4 Elliptic Curves and Modular Forms . . . . . . . . . . . . . . 31

5 Geometric Realization of Modular Forms of Higher Weight . . . 35

4 The Ramanujan Conjecture from $G L(2)$ to $G L(n) \ldots \ldots \ldots$

1 The Ramanujan Conjectures . . . . . . . . . . . . . . . . . . . . 39

2 Maass Forms of Weight Zero . . . . . . . . . . . . . . . . 45

3 Upper Bound for Fourier Coefficients and Eigenvalue Estimates . . 47

4 Eisenstein Series . . . . . . . . . . . . . . . . . . . . . . . . . . . 49

$5 \quad$ Eisenstein Series and Non-vanishing of $\zeta(s)$ on $\Re(s)=1 \quad \ldots \quad \ldots 51$

6 The Rankin-Selberg $L$-Function . . . . . . . . . . . . . . . 54

$7 \quad$ Poincaré Series for $S L_{2}(\mathbb{Z}) \quad \ldots \ldots \ldots \ldots$

8 Fourier Coefficients and Kloosterman Sums . . . . . . . . . 60

9 The Kloosterman-Selberg Zeta Function . . . . . . . . . . . . . . 64

10 Rankin-Selberg $L$-Functions for $G L_{n} \ldots \ldots \ldots$ 
5 The Circle Method . . . . . . . . . . . . . . . . . . . . 67

1 Introduction . . . . . . . . . . . . . . . . . 67

2 The Partition Function . . . . . . . . . . . . . . . . . 69

3 Waring's Problem . . . . . . . . . . . . . . . . 74

3.1 Schnirelmann Density . . . . . . . . . . . . 75

3.2 Schnirelmann Density and Waring's Problem . . . . . . . . 78

3.3 Proof of Linnik's Theorem . . . . . . . . . . . . . . . 80

4 Goldbach's Conjecture . . . . . . . . . . . . . . . . . . . . 87

$4.1 \quad$ Basic Lemmas . . . . . . . . . . . . . . . . . . . . . . . . . . . . . . . . . . .

4.2 Major Arcs . . . . . . . . . . . . . . . . . . . . . . 90

4.3 Application of Partial Summation . . . . . . . . . . . . 91

4.4 Primes in Arithmetic Progressions . . . . . . . . . . . 91

4.5 The Singular Series . . . . . . . . . . . . . . . . 92

4.6 The Minor Arcs Estimate Using GRH . . . . . . . . . . . 95

6 Ramanujan and Transcendence . . . . . . . . . . . . . . . . . 97

1 Nesterenko's Theorems . . . . . . . . . . . . . . . . . . . 97

2 Special Values of the $\Gamma$-Function at CM Points . . . . . . . . . . . 100

3 Special Values of Jacobi's Theta Series . . . . . . . . . . . . . . 101

4 The Rogers-Ramanujan Continued Fraction . . . . . . . . . . . . 102

5 Nesterenko's Conjectures . . . . . . . . . . . . . . . . 103

6 Special Values of the Riemann Zeta Function and $q$-Analogues . . 104

7 Arithmetic of the Partition Function . . . . . . . . . . . . . . . . . . 109

1 Ramanujan's Congruences . . . . . . . . . . . . . . . . . . . . 109

2 Higher Congruences . . . . . . . . . . . . . . . . . . . 113

3 Dyson's Ranks and Cranks . . . . . . . . . . . . . . . 114

4 Parity Questions .................. . 116

8 Some Nonlinear Identities for Divisor Functions . . . . . . . . . . . . 119

1 A Quadratic Relation Amongst Divisor Functions . . . . . . . . . 119

2 Quadratic Relations Amongst Eisenstein Series . . . . . . . . . . . 120

3 A Formula for the $\tau$-Function . . . . . . . . . . . . . . . 121

4 Derivatives of Modular Forms . . . . . . . . . . . . . . . . 122

5 Differential Operators and Nonlinear Identities . . . . . . . . . . . 124

6 Quasi-modular Forms . . . . . . . . . . . . . . . 125

7 Non-linear Congruences and Their Interpretation . . . . . . . . . 127

9 Mock Theta Functions and Mock Modular Forms _ . . . . . . . . . 129

1 Historical Introduction . . . . . . . . . . . . . . . . . . . . . . . 129

2 Ramanujan's Examples . . . . . . . . . . . . . . . . 130

3 The Work of Zwegers . . . . . . . . . . . . . . . . . . . 130

4 The Space of Mock Modular Forms . . . . . . . . . . . . . . . . . 132

5 Some Applications . . . . . . . . . . . . . . . . . . 133

10 Prime Numbers and Highly Composite Numbers . . . . . . . . . . 135

1 The Divisor Functions . . . . . . . . . . . . . . . . 135 
2 Ramanujan and the Prime Number Theorem . . . . . . . . . 138

3 Highly Composite Numbers . . . . . . . . . . . . . . . . . . . 141

4 Relation to the Six Exponential Conjecture . . . . . . . . . . 143

5 Counting Highly Composite Numbers . . . . . . . . . . . . . . . 144

6 Maximal Order of Divisor Functions and Other Arithmetic Functions . . . . . . . . . . . . . . . . . . . . . 145

7 Maximal Orders of Fourier Coefficients of Cusp Forms . . . . . 147

11 Probabilistic Number Theory . . . . . . . . . . . . . . . . . . . . . . 149

1 The Normal Order Method . . . . . . . . . . . . . . . . . . . 149

2 The Erdös-Kac Theorem . . . . . . . . . . . . . . . 150

3 The Hardy-Ramanujan-Type Theorem for the $\tau$-Function . . . . . 151

4 Non-abelian Generalizations of the Hardy-Ramanujan Theorem . . 153

12 The Sato-Tate Conjecture for the Ramanujan $\tau$-Function . . . . . 155

1 Introduction . . . . . . . . . . . . . . . 155

2 Weyl's Criterion . . . . . . . . . . . . . . . . . . 159

3 Wiener-Ikehara Tauberian Theorem . . . . . . . . . . . . 161

4 Weyl's Theorem for Compact Groups . . . . . . . . . . . . . . 163

5 Symmetric Power $L$-Series of Elliptic Curves . . . . . . . . . . 164

6 An Outline of the Proof of the Sato-Tate Conjecture . . . . . . 166

7 A Chebotarev-Sato-Tate Theorem and Generalizations . . . . . 168

8 Concluding Remarks . . . . . . . . . . . . . . . 170

Erratum to: The Ramanujan $t$-Function $\ldots \ldots \ldots \ldots$. . . . . . E1

References . . . . . . . . . . . . . . . . . . . . . 173

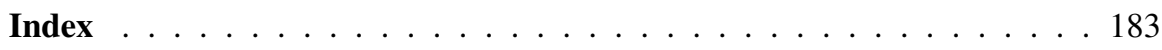

